\section{L'abus de substances volatiles}

L Baydala; Société canadienne de pédiatrie, comité de la santé des Premières nations, des Inuits et des Métis

L 'abus de substances volatiles, également connu sous le nom d'abus de substances inhalées, d'abus de solvants, de sniffing (ou snifage - reniflage à partir d'un contenant), de huffing (inhalation d'un linge imprégné) ou de bagging (inhalation dans un sac placé autour de la bouche et du nez), désigne l'inhalation délibérée d'une substance volatile pour altérer l'état mental. Par rapport aux autres formes de consommation de drogues, il touche souvent les enfants plus jeunes et traverse les frontières sociales et ethniques. L'abus de substances volatiles est un problème mondial particulièrement courant au sein des populations minoritaires et marginalisées, et il a une forte corrélation avec les déterminants sociaux de la santé (1). Le présent document de principes analyse les aspects cruciaux de l'abus de substances volatiles, fait ressortir de nouveaux renseignements et de nouvelles données à l'égard des enfants et adolescents autochtones et contient des recommandations en matière de traitement et de prévention. Il remplace le document de principes précédent de la Société canadienne de pédiatrie, publié en 1998 (2).

\section{L'ÉPIDÉMIOLOGIE}

L'absence de prise de conscience, l'opprobre social, les tendances en mutation et les différences régionales apparentes, de même que les différences dans la méthodologie des enquêtes, complique la déclaration exacte de l'épidémiologie de l'abus de substances volatiles. L'Enquête sur les toxicomanies au Canada (3), une enquête téléphonique aléatoire, révèle

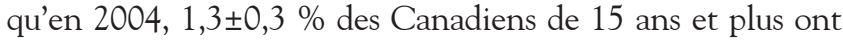
déclaré avoir consommé des substances volatiles pendant leur

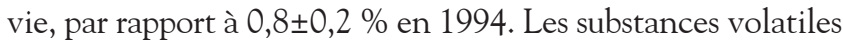
sont licites, peu coûteuses et faciles à obtenir, tous des éléments susceptibles d'expliquer le potentiel de consommation plus élevé chez les jeunes enfants. Dans une enquête menée en direct aux États-Unis (É.-U.) en 2007 (4), $1,1 \%$ des jeunes de 12 à 13 ans avaient consommé des substances volatiles au cours du mois précédent, et 66,3\% des répondants de 12 ans et plus étaient des enfants lorsqu'ils en avaient consommé pour la première fois. Notamment, 10,7 \% des répondants ont déclaré que les substances volatiles étaient leur première drogue. Les enquêtes canadienne et américaine excluaient les sans-abri et les détenus. La prévalence d'abus de substances volatiles pourrait donc être encore plus élevée. Les recherches varient également pour ce qui est de la catégorisation des diverses substances et de celles qui se qualifient comme des «substances volatiles ».

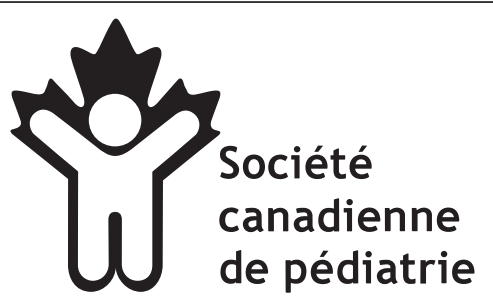

English on page 443

Résumé en page 443
Dans un sondage autoadministré auprès de jeunes des É.-U., moins d'élèves de la même cohorte de classe affirmaient s'être intoxiqués aux substances volatiles au cours des années subséquentes (5). Ces résultats peuvent s'expliquer parce que certains élèves qui consommaient des substances volatiles peuvent avoir quitté l'école ou oublié leurs expériences lorsqu'ils ont été interrogés plus tard. Il se peut aussi que les élèves plus âgés aient considéré l'abus de substances volatiles comme moins acceptable socialement et aient donc hésité davantage à le déclarer. La perception selon laquelle les substances volatiles sont une «drogue pour les p'tits » (tout comme l'utilisation du terme générique «sniffer de la colle », qui semble inoffensif) a été décrite. Cette perception pourrait expliquer les tendances épidémiologiques de l'abus de substances volatiles et mérite d'être prise en compte par les professionnels de la santé (6).

L'abus de substances volatiles est plus courant chez les décrocheurs, les personnes qui ont été négligées ou victimes de violence physique ou sexuelle, les détenus et les sans-abri, ainsi qu'au sein des communautés autochtones. $(1,7,8)$. Les différences entre les autres groupes ethniques et entre les sexes sont plus ambiguës. L'abus de substances volatiles est plus prévalent dans les collectivités rurales, de même que dans les collectivités isolées aux taux de chômage, de pauvreté et de violence élevés (1). Il est également corrélé avec un soutien familial limité ou un milieu familial déviant, de mauvais résultats scolaires, une mauvaise estime de soi et des tendances suicidaires, des troubles psychiatriques, la consommation d'autres drogues, des familles et des camarades qui consomment également $(1,8)$. L'abus de substances volatiles est un problème mondial étroitement relié aux déterminants de la santé. Les enfants et les adolescents provenant de divers contextes sociaux commencent à les consommer pour diverses raisons. Par exemple, dans les pays en développement, il permet souvent de soulager les symptômes de la faim (9).

\section{LA CONSOMMATION DE PRODUITS CHIMIQUES ET D'AUTRES PRODUITS}

Les substances volatiles sont des produits volatils diversifiés sur le plan pharmacologique, souvent sélectionnées pour leur faible coût, leur légalité et leur grande disponibilité, de même que pour leur capacité à induire l'euphorie rapidement. Leur type pharmacologique et leur mode de consommation ont permis de les regrouper en trois catégories : les hydrocarbures

Correspondance : Société canadienne de pédiatrie, 2305, boulevard St Laurent, Ottawa (Ontario) K1G 4J8, téléphone : 613-526-9397, télécopieur : 613-526-3332, Internet : www.cps.ca, www.soinsdenosenfants.cps.ca 
TABLEAU 1

Catégories, exemples et produits chimiques présents dans les substances volatiles les plus consommées

\begin{tabular}{|c|c|c|c|}
\hline Catégorie & Exemples & Produits chimiques & Autres termes \\
\hline $\begin{array}{l}\text { Hydrocarbures } \\
\text { aliphatiques, } \\
\text { aromatiques et } \\
\text { halogénés }\end{array}$ & $\begin{array}{l}\text { Fixatif pour cheveux, assainisseurs d'air, } \\
\text { déodorants } \\
\text { Carburants, y compris les briquets } \\
\text { Décapants et dissolvants, diluants pour peintures, } \\
\text { marqueurs, liquides correcteurs, colles et colles } \\
\text { caoutchoucs } \\
\text { Vernis, laques, résines, diluants à peinture-laque } \\
\text { Produits de nettoyage à sec, détachants, solvants } \\
\text { de dégraissage } \\
\text { Nettoyeurs en aérosol pour ordinateurs ou } \\
\text { produits électroniques } \\
\text { Aérosols de cuisson à l'huile végétale } \\
\text { Anesthésiques médicaux }\end{array}$ & $\begin{array}{l}\text { Butane, propane, fluorocarbones } \\
\text { Essence, propane, benzène, butane } \\
\text { Trichloroéthane, trichloroéthylène, toluène, } \\
\text { hexane, acétone, chlorure de méthylène, } \\
\text { acétate d'éthyle } \\
\text { Benzène, xylène } \\
\text { Trichloroéthane, tétrachloroéthylène, xylène } \\
\text { Méthoxyméthane, hydrofluorocarbones, } \\
\text { hydrocarbures } \\
\text { Hydrocarbures } \\
\text { Oxyde de diéthyle, halothane, enflurane, } \\
\text { chlorure d'éthyle }\end{array}$ & Colle, essence, air blast \\
\hline Protoxyde d'azote & $\begin{array}{l}\text { Crème fouettée en aérosol, bonbonnes } \\
\text { d'hélium pour ballons, anesthésiques }\end{array}$ & Protoxyde d'azote & $\begin{array}{l}\text { Gaz hilarant, shoot the breeze, } \\
\text { whippets, buzz bomb }\end{array}$ \\
\hline Nitrites d'alkyle volatils & $\begin{array}{l}\text { Médicaments contre l'angine, « parfums } \\
\text { d'ambiance », nettoyeurs de tête de } \\
\text { magnétoscope, produits synthétisés }\end{array}$ & $\begin{array}{l}\text { Nitrites de pentyle, nitrites de butyle, nitrite de } \\
\text { cyclohexyle, nitrite de propyl, autres nitrites }\end{array}$ & $\begin{array}{l}\text { Pearls, boppers, snappers, } \\
\text { poppers, rush, bolt, quicksilver, } \\
\text { aroma of men, hardware, locker } \\
\text { room, thrust }\end{array}$ \\
\hline
\end{tabular}

aliphatiques, aromatiques ou halogénés, le protoxyde d'azote et les nitrites d'alkyle volatils $(10,11)$ (tableau 1). Les substances les plus déclarées aux centres antipoison des É.-U. entre 1996 et 2001 sont l'essence (41\%), la peinture (13\%), le propane ou le butane $(6 \%)$, les assainisseurs d'air $(6 \%)$ et la formaline (5\%). La majorité des décès liés aux substances volatiles étaient causés par l'essence (45\%), les assainisseurs d'air (26\%) et le propane ou le butane (11\%) (12).

\section{LES EFFETS IMMÉDIATS}

Pour « sniffer » ou « renifler », il faut l'inhalation directe des vapeurs, l'inhalation dans un sac de plastique ou de papier, l'inhalation d'un linge imprégné de la substance et placé sur la bouche ou le nez, l'inhalation d'assainisseur d'air en aérosol et la vaporisation directe de nettoyants en aérosol dans la bouche ou le nez $(10,11)$. Souvent, il faut effectuer plusieurs inhalations profondes pour obtenir des effets euphoriques optimaux. En raison de l'absorption pulmonaire rapide et de la solubilité des lipides, le cerveau est vite touché (13). La plus forte concentration de substances volatiles provient de l'inhalation dans un sac et de l'inhalation d'un linge imprégné. C'est pourquoi ce sont les méthodes privilégiées (14).

Les effets initiaux sont similaires à ceux des anesthésiques : une stimulation, une désinhibition et une euphorie. Ces sensations peuvent être suivies d'hallucinations, puis d'une dépression générale, y compris un empâtement de la parole et une perturbation de la démarche, des étourdissements, une désorientation et une somnolence ou le sommeil dans un délai de quelques secondes à quelques minutes (13). Pendant l'inhalation dans un sac, la réinhalation potentialise la consommation par une hypercapnie et une hypoxie (15). La somnolence et les céphalées peuvent persister pendant des heures en raison de l'intoxication résiduelle. La fatigue et le sommeil limitent souvent le degré d'intoxication (13). Les substances volatiles s'accompagnent de toute une série d'effets sur la libération des neurotransmetteurs et des récepteurs et s'associent à quelques actions cellulaires semblables à celles d'autres dépresseurs comme les benzodiazépines, l'alcool et les barbituriques (16).
Les nitrites d'alkyle volatils, comme ceux qui sont indiqués pour le traitement de l'angine, ont des effets vasodilatateurs qui entraînent une hypotension et une syncope, de même que des sensations de chaleur et de rougeurs. Les « poppers » et leurs sensations s'associent aux bars et aux activités sexuelles. Ils sont également consommés pour leur effet de relaxation du sphincter et d'engorgement pénien chez les jeunes hommes en prévision de relations sexuelles anales, ce qui les lie à un accroissement de la transmission du VIH (17). Il est indiqué que divers types de substances volatiles provoquent une engelure ou une brûlure du visage et des voies aérodigestives supérieures (18).

On a signalé au moins un cas d'arrêt respiratoire attribuable à une dépression du système nerveux central, et il est postulé que le refroidissement subit du larynx (par vaporisation directe d'aérosols) serait responsable d'une dépression vagale fatale (19). Probablement à cause des arythmies cardiaques, la mort subite après inhalation volontaire est la principale cause de décès chez les consommateurs de substances volatiles. Les substances volatiles perturbent la propagation électrique du myocarde, un effet amplifié par l'hypoxie, ce qui accroît le risque d'arythmie. Elles sensibilisent également le cœur à l'adrénaline; une mort subite après inhalation volontaire peut se produire lorsque le consommateur est surpris pendant l'inhalation (s'il est pris en flagrant délit, par exemple) ou s'il a des hallucinations saisissantes (19). Les décès et les traumatismes secondaires à une consommation aiguë découlent souvent de comportements dangereux (par exemple, noyade, chute ou saut, brûlures ou hypothermie) attribuables à la désinhibition et aux sentiments d'invincibilité, tandis que l'aspiration et la suffocation, surtout pendant l'inhalation dans un sac, peuvent également être fatales (10).

\section{LES EFFETS À LONG TERME}

Il est démontré que la consommation chronique a des effets neurologiques et neuropsychologiques radicaux et irréversibles, probablement causés par les dommages à la myéline et aux membranes neuronales attribuables aux produits chimiques lipophiles (15). Plus concentrés dans les tissus nerveux que 
dans le sang, ces produits chimiques peuvent être responsables d'une atrophie corticale et de lésions visibles à la neuroimagerie. On sait que les substances volatiles entraînent des dysfonctions du tronc cérébral et toute une série de déficits moteurs, cognitifs et sensoriels (20). Les signes peuvent inclure l'irritabilité, les tremblements, l'ataxie, le nystagmus, l'empâtement de la parole, une diminution de l'acuité visuelle et la surdité (13).

L'abus de substances volatiles peut entraîner une myocardiopathie aux modifications électrocardiographiques distinctes (21). Il peut provoquer une dyspnée, des anomalies emphysémateuses et d'autres anomalies pulmonaires débilitantes, une acidose tubulaire distale et une hépatite (22). Les hydrocarbures peuvent entraîner une toxicité de la moelle osseuse (responsable d'une anémie aplasique et d'une leucémie) (23), tandis que les nitrites volatils participent à l'atteinte immunitaire et à la réplication du VIH et du sarcome de Kaposi, de même qu'à la création de nitrosamine cancérigène et de méthémoglobine comme métabolites (13).

Les femmes qui sont exposées professionnellement à des solvants ont plus de troubles menstruels, tandis que la prééclampsie et les avortements spontanés sont plus courants chez les femmes enceintes qui consomment des substances volatiles (24). Les effets tératogènes secondaires à l'exposition de la mère aux substances volatiles peuvent donner lieu à un « syndrome foetal d'inhalation aux solvants " associé à des anomalies neurologiques congénitales, y compris une microcéphalie et des atteintes cognitives (25). Les signes de sevrage néonatal incluent un cri aigu ainsi qu'une perturbation du sommeil et de l'alimentation (26).

L'abus de substances volatiles s'associe à de mauvais résultats scolaires, à des comportements criminels, à la consommation d'autres drogues, à une mésadaptation sociale, à une mauvaise estime de soi et à des tendances suicidaires $(1,4)$. La causalité de ces phénomènes n'est pas établie puisque ceux-ci peuvent être antérieurs ou directement reliés à l'abus de substances volatiles.

\section{LA DÉTECTION}

Les signes d'abus de substances volatiles sont souvent plus subtils que ceux de la consommation d'autres drogues. Les effets sont ressentis rapidement et disparaissent vite (11), et seule une petite quantité de la substance suffit. De plus, les produits consommés sont généralement licites, ce qui rend leur achat et leur rangement moins ostensibles. Les consommateurs peuvent avoir des provisions anormalement importantes de substances volatiles, parfois rangées dans des endroits atypiques (tels que sous le lit d'un enfant). Les consommateurs chroniques peuvent présenter des signes physiques clairs : haleine particulière qui peut persister pendant des heures, taches, peintures, poudre scintillante ou odeur sur la peau ou les vêtements. La présentation peut également inclure une sécheresse périorale ou une pyodermite, une «éruption des inhalateurs » (parfois jaunâtre chez les consommateurs de nitrites) ou même des engelures ou des brûlures sur le visage, dans la bouche, le nez ou le passage œsopharyngé, parfois accompagnées d'un œdème secondaire

\section{TABLEAU 2}

Outil de dépistage CRAFFT (d'après l'acronyme anglais)

C As-tu déjà monté dans une voiture conduite par quelqu'un (toi y compris) qui était « high » ou qui avait consommé de l'alcool ou des drogues?

R T'arrive-t-il de consommer de l'alcool ou des drogues pour te détendre, mieux t'accepter ou t'intégrer?

A T'arrive-t-il de consommer de l'alcool ou des drogues lorsque tu es seul?

F T'arrive-t-il d'oublier des choses que tu as faites pendant que tu consommais de l'alcool ou des drogues?

F Les membres de ta famille ou tes amis t'ont-ils déjà dit que tu devrais réduire ta consommation d'alcool ou de drogues?

T T'est-il déjà arrivé de t'attirer des ennuis pendant que tu consommais de l'alcool ou des drogues?

Au moins deux « oui » indiquent un problème potentiel de consommation et la nécessité d'une évaluation plus approfondie. Traduit avec l'autorisation de la référence 51. (C) Association médicale canadienne.

des lèvres, de l'oropharynx et de la trachée (13). Des manifestations d'atteinte neuropsychologique (comme la confusion, l'instabilité émotive ou l'irritabilité) et de toxicité pulmonaire (y compris la respiration sifflante, l'emphysème et la dyspnée) peuvent constituer d'autres signes évidents $(13,15,27)$. On peut déceler une grave toxicité en cas de dépression du système nerveux central plus prononcée, telle qu'une ataxie, des tremblements et un nystagmus $(27,28)$. Les amis et la famille peuvent soupçonner une consommation importante s'ils remarquent une mauvaise hygiène, une perte de poids, de la fatigue, des saignements de nez, une conjonctivite, une faiblesse musculaire, des nausées, une apathie, un manque d'appétit et des troubles gastrointestinaux, des modifications de l'assiduité scolaire ou des changements psychologiques ou psychiatriques $(27,28)$. Une forte prévalence de troubles des humeurs, de troubles anxieux et de troubles de la personnalité est corrélée avec l'abus de substances volatiles, et il faut en tenir compte tant pour le dépistage que pour le traitement subséquent.

On peut procéder à une analyse des métabolites urinaires pour déceler la présence de certains solvants (benzène, toluène, xylène et solvants chlorés) afin de s'assurer du respect du traitement, mais en général, un dépistage urinaire ordinaire et d'autres tests de laboratoire ne permettent pas de déceler l'abus de substances volatiles $(10,29)$. Même si c'est peut-être l'outil le plus précieux pour déceler un abus de substances volatiles, les professionnels de la santé procèdent rarement au dépistage (30). Ils sont avisés d'envisager l'utilisation d'un outil de dépistage comme le questionnaire CRAFFT (tableau 2) et d'en connaitre les bonnes techniques d'administration, y compris des entrevues privées et la participation de la famille par l'obtention d'antécédents plus complets (30-32). De plus, la famille, les camarades, les enseignants, les conseillers et les autres intervenants devraient être mis au courant des signes d'abus de substances volatiles et du mode d'aiguillage des enfants et des adolescents susceptibles d'y être vulnérables.

Le dépistage généralisé des jeunes et des détenus a favorisé un aiguillage réussi vers des programmes thérapeutiques (33), tandis que le projet pilote des É.-U., intitulé Screening, Brief Intervention, and Referral to Treatment (SBIRT), a établi que 22,7\% des 459599 patients étaient 
positifs à une certaine participation ou à un risque de consommation de drogues. Un aiguillage précoce vers un programme thérapeutique a favorisé une amélioration importante de l'état physique, mental et social des personnes touchées (34).

\section{L'INTERVENTION ET LE TRAITEMENT}

Les consommateurs de substances volatiles ne demanderont pas nécessairement d'aide médicale, sauf en cas de blessure connexe ou de grave maladie. Il n'existe pas de traitement efficace contre une intoxication aiguë aux substances volatiles et contre le sevrage, sauf des soins de soutien d'une extrême vigilance (35). La prise en charge peut inclure la prescription d'antiarythmiques ou de béta-bloquants pour stabiliser le myocarde et éviter une surstimulation par les catécholamines. Il faut éviter les sympathomimétiques (19). L'hypotension est courante, et il faut surveiller l'état cardiopulmonaire, de même que l'état mental (11,35). Il faudra peut-être décontaminer la peau et les vêtements (35). Il faut obtenir les antécédents du patient auprès de sa famille et de ses camarades, sans compter qu'il est essentiel d'établir son état neurologique et important de procéder à des examens de laboratoire pour dépister les atteintes hépatique, rénale et cardiaque (13). Les symptômes de sevrage peuvent influer sur le traitement, car il en existe diverses manifestations, telles que des nausées, une anorexie, une sudation, des tics, des troubles du sommeil et une instabilité émotive marquée $(13,28,36)$.

Les consommateurs de substances volatiles sont plus jeunes et présentent une plus grande dysfonction sociale que les consommateurs d'autres drogues. Par conséquent, il faut prévoir des programmes thérapeutiques propres aux substances volatiles, qui incluent une période de détoxication, le recours à un système de défense camarades-patient, le développement des forces et des habiletés et une transition pertinente de retour dans la collectivité (37). Il faut envisager la présence d'autres dépendances ou d'un trouble psychiatrique ou physique et traiter les pathologies concomitantes. Un traitement rapide, rendu possible par une détection précoce grâce à un dépistage généralisé, et des périodes de traitement prolongées améliorent les issues $(35,38)$. Les familles de consommateurs de substances volatiles sont souvent dysfonctionnelles, et une thérapie familiale, accompagnée d'un contrat social avec la famille comportant un renforcement parental et le respect obligatoire de règles de comportement par le jeune, peut également se révéler efficace pour le traitement. Enfin, les professionnels de la santé devraient se rendre disponibles pendant et après le traitement, afin de favoriser et de faciliter le respect des programmes sur les compétences parentales, sur le soutien des parents et sur les habiletés sociales des jeunes, susceptibles de réduire le risque de rechute (39).

\section{LA PRÉVENTION}

Presque sans équivoque, la prévention est considérée comme la stratégie la plus efficace pour lutter contre l'abus de substances volatiles. Les méthodes préventives doivent viser à en réduire l'acceptabilité sociale, être complètes et appliquées en milieu communautaire, faire participer non seulement les consommateurs et les membres de leur famille, mais également leurs camarades, les écoles et les détaillants (9).

La participation des producteurs de produits licites aux interventions de prévention de l'abus de substances volatiles a eu des résultats mitigés. Dans trois États américains, la législation a été abandonnée à cause de difficultés à trouver des additifs pertinents en vue de rendre les substances déplaisantes pour les consommateurs, et l'étiquetage obligatoire des produits chimiques utilisés dans les substances volatiles a permis de repérer plus facilement les produits susceptibles d'être inhalés (40). L'Australie a obtenu un certain succès en remplaçant les composants nuisibles des produits, surtout pour respecter des exigences environnementales (41). Les détaillants et les fabricants peuvent également s'associer à l'éducation préventive (40).

Les préadolescents sont vulnérables à l'abus de substances volatiles. C'est pourquoi une éducation préventive probante doit être instaurée tôt dans les écoles primaires, pour s'assurer que les messages soient transmis avant, et non pendant que le jeune commence à consommer des substances volatiles (6). Il est établi que de brèves interventions en cabinet, y compris une rencontre de cinq à dix minutes pour souligner les risques liés à la consommation de drogues et d'alcool, sont efficaces pour réduire la consommation d'alcool, de marijuana et de tabac (42). Cependant, des stratégies similaires ne se sont pas révélées efficaces à l'égard de l'abus de substances volatiles, car la perception de méfait des produits est plus fortement corrélée aux réseaux sociaux qu'aux futures intentions d'en consommer (43). Ainsi, la disponibilité et l'abus de substances volatiles par les camarades sont liées à leur consommation, de nombreux jeunes déclarant en consommer au domicile d'amis et sur les terrains de l'école (44). De plus, l'abus de substances volatiles est relié à la pauvreté, à la faim, à la maladie, à un faible niveau de scolarité, au chômage, à l'ennui et aux sentiments de désespoir (45). Il est donc clair que la prévention doit également tenir compte de l'influence des facteurs sociaux, y compris les déterminants sociaux de la santé. Des mesures intersectorielles, qui incluent des partenariats entre les organismes communautaires, le secteur privé et le gouvernement, s'imposent pour diffuser l'information et assurer une éducation sur l'abus de substances volatiles, ainsi que pour mettre au point des politiques favorisant la prévention $(40,46)$.

\section{LES CONSIDÉRATIONS À L'ÉGARD DE ENFANTS ET DES ADOLESCENTS AUTOCHTONES}

Plusieurs études (47) font état de taux plus élevés d'abus de substances volatiles au sein de certaines populations autochtones (notamment les communautés inuites et des Premières nations, mais aucune étude ne traite spécifiquement des Métis). Il est toutefois important de souligner qu'à l'instar de la consommation d'autres drogues, ces taux plus élevés s'expliquent probablement par la situation socioéconomique et ne sont pas attribuables à «l'ethnicité » de ces populations $(45,48)$. Dans certaines communautés, on 
signale que l'abus de substances volatiles est ignoré ou normalisé, bien que les reportages médiatiques aient peutêtre biaisé les perceptions quant à sa prévalence (49). Les écarts de santé, l'accès réduit aux services et des facteurs socioéconomiques peuvent contribuer à ces taux plus élevés. Les contextes sociaux et sanitaires uniques, ainsi que la nature généralisée de l'abus de substances volatiles dans certaines communautés, ont suscité la création de programmes thérapeutiques adaptés à la culture.

Le Programme national de lutte contre l'abus de solvants chez les jeunes (PNLASJ) a été créé en collaboration avec Santé Canada et les communautés des Premières nations afin d'accroître la capacité des jeunes à affronter l'adversité et à obtenir du soutien grâce à une plus forte identité culturelle (50). Le traitement à long terme en résidence (pour créer un lieu sécuritaire distinct de la communauté de résidence) vise à intégrer des éléments spirituels à la guérison, ce qui constitue un volet essentiel du succès du programme. Les clients sont incités à trouver et à utiliser des appuis dans leur communauté et dans leur école, et la participation de la famille est exigée. La réussite du programme continue de croître parce que celui-ci met en œuvre des services d'approche proactifs en vue de prévenir l'abus de substances volatiles, qu'il tient compte de la consommation d'autres drogues dans le traitement et qu'il continue d'intégrer la recherche aux résultats des efforts thérapeutiques et préventifs.

\section{CONCLUSION ET RECOMMANDATIONS}

La Société canadienne de pédiatrie est très préoccupée par l'abus de substances volatiles chez les enfants et les adolescents et est d'avis qu'il faut en faire davantage pour comprendre et combattre cette pratique dangereuse.

Les professionnels de la santé doivent prendre conscience des principaux facteurs liés à l'abus de substances volatiles, y compris les suivants :

- son épidémiologie et ses tendances;

- ses signes et symptômes, ses effets et ses dangers et le traitement des cas aigus;

- les techniques de dépistage;

- la disponibilité des ressources locales de prévention, d'intervention et de traitement.

Les professionnels de la santé doivent être des ambassadeurs de l'éducation et travailler avec les enfants et les adolescents, les parents, les écoles, les enseignants, les médias et les décideurs, notamment à l'égard des éléments suivants :

- les types et les exemples de produits chimiques utilisés;

- les signes et les dangers liés à l'abus de substances volatiles;

- l'intervention et le traitement : ce qu'il faut faire si on soupçonne quelqu'un de consommer;

- la lutte contre les idées fausses : préciser que même une consommation épisodique est dangereuse.

Les professionnels de la santé devraient tirer profit de leurs connaissances, de leurs expériences et de leurs relations dans la collectivité pour jouer les rôles suivants :

- orienter la création d'un réseau de soins sanitaires et communautaires pour les consommateurs de substances volatiles;

- orienter les politiques au sujet de la prévention, de l'éducation et du traitement de l'abus de substances volatiles;

- s'assurer que les déterminants sociaux de la santé qui influent sur l'abus de substances volatiles sont compris et pris en compte dans les politiques;

- prôner le remplacement des substances dangereuses et psychoactives contenues dans les produits courants par des substances moins nocives;

- préconiser et mener des recherches qui permettent de mieux comprendre l'abus de substances volatiles, y compris l'épidémiologie et des stratégies préventives et thérapeutiques efficaces qui tiennent compte des déterminants sociaux de la santé, surtout au sein des populations autochtones, et qui sont axées sur les populations métisses, inuites et des Premières nations. Les services de santé et les autorités sanitaires doivent s'assurer de l'accès à des programmes thérapeutiques familiaux efficaces, y compris des traitements à long terme en résidence lorsqu'ils sont nécessaires.

REMERCIEMENTS : L'auteure tient à remercier Ellison Richmond, adjointe de recherche, pour son apport important au présent document. La Société canadienne de pédiatrie remercie également le docteur Michael Rieder, président du comité de la pharmacologie et des substances dangereuses, qui a révisé le présent document de principes pour le compte de son comité.

\section{RÉFÉRENCES}

1. Kozel N, Sloboda Z, De La Rosa M, éd. Epidemiology of inhalant abuse: An international perspective (NIDA Research Monograph 148). www.nida.nih.gov/pdf/monographs/148.pdf (consulté le $1^{\text {er }}$ mars 2010).

2. Société canadienne de pédiatrie, comité de la santé des Premières nations et des Inuits [auteur principal : M Tenenbein]. L'intoxication aux substances inhalées. Paediatr Child Health 1998;3:129-33.

3. Adlaf EM, Begin P, Sawka E, éd. Canadian Addiction Survey (CAS): A national survey of Canadians' use of alcohol and other drugs: Prevalence of use and related harms: Detailed report. Ottawa: Canadian Centre on Substance Abuse, 2005. <www.ccsa.ca/2005\%20CCSA\%20Documents/ ccsa-004028-2005.pdf> (consulté le $1^{\text {er }}$ mars 2010).

4. Substance Abuse and Mental Health Services Administration. Results from the 2007 National Survey on Drug Use and Health: National findings <www.oas.samhsa.gov/NSDUH/2k7NSDUH/ $2 \mathrm{k} 7$ results.cfm $>$ (consulté le $1^{\mathrm{er}}$ mars 2010).

5. Johnston LD, O'Malley PM, Bachman JG, Schulenberg JE. Monitoring the future: National survey results on drug use, 1975-2007: Volume II: College students and adults ages 19-45. Bethesda: National Institute on Drug Abuse, 2007.

6. Wu LT, Ringwalt CL. Inhalant use and disorders among adults in the United States. Drug Alcohol Depend 2006;85:1-11.

7. Sakai JT, Hall SK, Mikulich-Gilbertson SK, Crowley TJ. Inhalant use, abuse, and dependence among adolescent patients: Commonly comorbid problems. J Am Acad Child Adolesc Psychiatry 2004;43:1080-8.

8. Medina-Mora ME, Real T. Epidemiology of inhalant use. Curr Opin Psychiatry 2008;21:247-51.

9. Dell CA, Beauchamp T; Centre canadien de lutte contre l'alcoolisme et les toxicomanies. FAQ sur l'abus de solvants volatils chez les jeunes, 2006. <www.ccsa.ca/2006\%20CCSA\%20Documents/ccsa-011327-2006.pdf> (consulté le $1^{\mathrm{er}}$ mars 2010). 
10. Williams JF, Storck M; American Academy of Pediatrics Committee on Substance Abuse, American Academy of Pediatrics on Native American Child Health. Inhalant abuse. Pediatrics 2007;119:1009-17.

11. Lorenc JD. Inhalant abuse in the pediatric population: A persistent challenge. Curr Opin Pediatr 2003;15:204-9.

12. Spiller HA. Epidemiology of volatile substance abuse (VSA) cases reported to US poison centers. Am J Drug Alcohol Abuse 2004;30:155-65.

13. Brouette T, Anton R. Clinical review of inhalants. Am J Addict 2001;10:79-94.

14. Crocetti M. Inhalants. Pediatr Rev 2008;29:33-4.

15. Meadows R, Verghese A. Medical complications of glue sniffing. South Med J 1996;56:455-62.

16. Balster RL. Neural basis of inhalant abuse. Drug Alcohol Depend 1998;51:207-14.

17. Romanelli F, Smith KM, Thornton AC, Pomeroy C.

Poppers: Epidemiology and clinical management of inhaled nitrite abuse. Pharmacotherapy 2004;24:69-78.

18. Albright JT, Lebovitz BL, Lipson R, Luft J. Upper aerodigestive tract frostbite complicating volatile substance abuse. Int J Pediatr Otorhinolaryngol 1999;49:63-7.

19. Shepherd RT. Mechanism of sudden death associated with volatile substance abuse. Hum Toxicol 1989;8:287-91.

20. Rosenberg NL, Grigsby J, Dresibach J, Busenbark D, Grigsby P. Neuropsychologic impairment and MRI abnormalities associated with chronic solvent abuse. J Toxicol Clin Toxicol 2002;40:21-34

21. Vural M, Ogel K. Dilated cardiomyopathy associated with toluene abuse. Cardiology 2006;105:158-61.

22. Marjot R, Mcleod AA. Chronic non-neurological toxicity from volatile substance abuse. Hum Toxicol 1989;8:301-6.

23. Rinsky RA. Benzene and leukemia: An epidemiologic risk assessment. Environ Health Perspect 1989;82:189-91.

24. Jones HE, Balster RL. Inhalant abuse in pregnancy. Obstet Gynecol Clin North Am 1998;25:153-67.

25. Lin $\mathrm{H}$, Liu C, Jow G, Tang C. Toluene disrupts synaptogenesis in cultured hippocampal neurons. Toxicol Lett 2009;184:90-6.

26. Tenenbein M, Casiro OG, Seshia MMK, Debooy VD. Neonatal withdrawal from maternal volatile substance abuse. Arch Dis Child Fetal Neonatal Ed 1996;74:F204-7.

27. Ridenour TA. Inhalants: Not to be taken lightly anymore. Curr Opin Psychiatry 2005;18:243-7.

28. Anderson CE, Loomis GA. Recognition and prevention of inhalant abuse. Am Family Physician 2003;68:869-74.

29. Broussard LA. The role of the laboratory in detecting inhalant abuse. Clin Lab Sci 2000;13:205-9.

30. Dias PJ. Adolescent substance abuse. Assessment in the office. Pediatr Clin North Am 2002;49:269-300.

31. Knight JR, Sherritt L, Shrier LA, Harris SK, Chang G. Validity of the CRAFFT substance abuse screening test among adolescent clinic patients. Arch Pediatr Adolesc Med 2002;156:607-14.

32. Kulig JW; American Academy of Pediatrics Committee on Substance Abuse. Tobacco, alcohol, and other drugs: The role of the pediatrician in prevention, identification, and management of substance abuse. Pediatrics 2005;115:816-21.

33. Poulin C; Centre canadien de lutte contre l'alcoolisme et les toxicomanies. Politiques et programmes de réduction des méfaits pour les jeunes, $2006<$ www.ccsa.ca/2006\%20CCSA\%20Documents/ccsa11340-2006.pdf> (consulté le $1^{\text {er }}$ mars 2010).
34. Madras BK, Compton WM, Avula D, Stegbauer T, Stein JB, Clark HW. Screening, brief interventions, referral to treatment (SBIRT) for illicit drug and alcohol use at multiple healthcare sites: Comparison at intake and 6 months later. Drug Alcohol Depend 2009;99:280-95.

35. Muller AA, Muller GF. Inhalant abuse. J Emerg Nurs 2006;32:447-8.

36. Keriotis AA, Updahyaya HP. Inhalant dependence and withdrawal symptoms. J Am Acad Child Adolesc Psychiatry 2000;39:679-80.

37. Jumper-Thurman P, Plested B, Beauvais F. Treatment strategies for volatile solvent abusers in the United States. NIDA Res Monogr 1995;148:250-9.

38. Dell CA, Ogborne A, Begin P et coll.; Centre canadien de lutte contre l'alcoolisme et les toxicomanies. Conception d'un programme de traitement en résidence de l'abus de solvants chez les jeunes : un examen du rôle de la durée du programme et de la durée du séjour des clients, 2003. <www.ccsa.ca/2003\%20and\%20earlier\%20CCSA $\% 20$ Documents/extl-003883-2003.pdf> (consulté le $1^{\text {er }}$ mars 2010).

39. Jaffe SL. Treatment and relapse prevention for adolescent substance abuse. Pediatr Clin North Am 2002;49:345-52.

40. Liss BI. Government, trade and industry and other preventative responses to volatile substance abuse. Hum Toxicol 1989;8:327-30.

41. MacLean SJ, d'Abbs PHN. Will modifying inhalants reduce volatile substance misuse? A review. Drugs: Education, Prevention, and Policy 2006;13:453-39.

42. Toumbourou JW, Stockwell T, Neighbors C, Marlatt GA, Sturge J, Rehm J. Interventions to reduce harm associated with adolescent substance abuse. Lancet 2007;369:1391-401.

43. Perron BE, Howard MO. Perceived risk of harm and intentions of future inhalant use among adolescent inhalant users. Drug Alcohol Depend 2008;97:185-9.

44. Johnson K, Holder H, Ogilvie K et coll. A community prevention intervention to reduce youth from inhaling and ingesting harmful legal products. J Drug Educ 2007;37:227-47.

45. Lemstra M, Neudorf C, Nannapeneni U, Bennett N, Scott C, Kershaw T. The role of economic and cultural status as risk indicators for alcohol and marijuana use among adolescents. Paediatr Child Health 2009;14:225-30.

46. de Carvalho D. Case study: Intersectoral action to reduce petrol sniffing in remote communities of Central Australia. Agence de la santé publique du Canada, 2007.

47. Coleman H, Charles G, Collins J. Inhalant use by Canadian Aboriginal youth. J Child Adolesc Substance Abuse 2001;10:1-20.

48. Edwards RW, Stanley L, Plested BA, Marquart BS, Chen J, Jumper-Thurman P. Disparities in young adolescent inhalant use by rurality, gender, and ethnicity. Subst Use Misuse 2007;42:643-70.

49. Denov M, Campbell K. Casualties of Aboriginal displacement in Canada: Children at risk among the Innu of Labrador. Refuge 2002;20:21-33.

50. Programme national de lutte contre l'abus de solvants chez les jeunes. Santé Canada, 2005. <www.hc-sc.gc.ca/fniah-spnia/substan/ads/nysappnlasj-fra.php> (consulté le $1^{\text {er }}$ mars 2010).

51. Leslie K. Leslie K. Youth substance use and abuse: Challenges and strategies for identification and intervention. CMAJ 2008;178:145-8 (Ces travaux sont protégés par droit d'auteur, et cette traduction était autorisée par le Journal de l'Association médicale canadienne [www.cmaj.ca] et Access Copyright. Toute modification de son contenu ou copie sous quelque forme que ce soit est interdite, sous réserve d'une autorisation par la loi.)

\section{COMITÉ DE LA SANTÉ DES PREMIÈRES NATIONS, DES INUITS ET DES MÉTIS}

Membres : Docteurs William Abelson (représentant du conseil), Prince George (Colombie-Britannique); Anna Banerji, Toronto (Ontario); Lola Baydala, Edmonton (Alberta); Heidemarie Schröter, Calgary (Alberta); Sam Wong (président), Edmonton (Alberta)

Représentants : Mesdames Debbie Dedam-Montour (Organisation nationale des représentants indiens et inuits en santé communautaire); Carolyn Harrison (Santé Canada, Direction générale de la santé des Premières nations et des Inuits); Kathy Langlois (Santé Canada, Direction générale de la santé des Premières nations et des Inuits); Veronica Matthews (Association des infirmières et infirmiers autochtones du Canada); Heather McCormack (Santé Canada, Direction générale de la santé des Premières nations et des Inuits); docteure Kelly Moore (American Academy of Pediatrics, comité de la santé des enfants autochtones américains); mesdames Rena Morrison (Assemblée des Premières nations, secteur social et de la santé); Anna Claire Ryan (Inuit Tapiriit Kanatami); Barbara van Haute (Ralliement national des Métis)

Conseiller : Docteur Kent Saylor, Montréal (Québec)

Auteure principale : Docteure Lola Baydala, Edmonton (Alberta)

$\overline{\text { Les recommandations contenues dans le présent document ne sont pas indicatrices d'un seul mode de traitement ou d'intervention. Des }}$ variations peuvent convenir, compte tenu de la situation. Tous les documents de principes et les articles de la Société canadienne de pédiatrie sont régulièrement évalués, révisés ou supprimés, au besoin. Consultez la zone « Documents de principes » du site Web de la SCP (www.cps.ca/Francais/publications/Enonces.htm) pour en obtenir la version la plus à jour. 